\title{
Numerical study of the lean premixed PRECCINSTA burner with hydrogen enrichment
}

\author{
Giovanni Filomeno ${ }^{1, *}$, Tommaso Capurso ${ }^{1, * *}$, Marco Torresi ${ }^{1, * * *}$, and Giuseppe \\ Pascazio $^{1, * * * *}$ \\ ${ }^{1}$ Dipartimento di Meccanica, Matematica e Management, Politecnico di Bari, Via Re David, 20070125 \\ Bari, Italy
}

\begin{abstract}
Hydrogen combustion is one of the most promising solution to achieve a global decarbonization in power production and transports. Pure hydrogen combustion is far from becoming a standard but, during the energy transition, hydrogen co-firing can be a feasible and economically attractive shortterm measure. The use of hydrogen blending gives rise to several issues related to flashback, NOx emissions and thermo-acoustic instabilities. To improve the understanding of the effect of hydrogen enrichment, herein a numerical analysis of lean premixed hydrogen enriched flames is performed by means of $3 \mathrm{D}$ unsteady CFD simulations. The numerical model has been assessed against experimental results for both cold and reacting flows in terms of velocity profile (average) and flame shape (mean $\mathrm{OH}^{*}$ radical fields). The burner under investigation is the swirl stabilized PRECCINSTA studied at the Deutsches Zentrum für Luft-und Raumfahrt (DLR). The DLR's researchers have shown the effect of hydrogen addition on the flame topology and combustion instabilities at various operating conditions in terms of thermal power, equivalence ratio and $\mathrm{H}_{2}$ volume fraction. Simulations are in good accordance with experimental data both in terms of velocity and temperature profiles. The numerical model provides a qualitative estimation of the flame shape.
\end{abstract}

\section{Introduction}

In the past decades, natural gas power generation represented one of the main solutions for the coal-to-gas transition. The replacement of coal with natural gas avoided $95 \mathrm{Mt} / \mathrm{year}$ of CO2 emissions [1] but, nowadays, this is no longer sufficient. In 2014, the European Commission proposed a reduction of Greenhouse Gases emissions by $40 \%$ with respect to the 1990 levels until 2030 [2].

Green hydrogen combustion can represent a viable solution to reach the decarbonization of power generation industry since it is a carbon-free energy vector for combustion and energy-storing. Many researchers are currently studying the effect of hydrogen addition in natural gas-fuelled turbine with the aim of reaching $100 \%$ hydrogen combustion until 2030 [3].

\footnotetext{
*e-mail: giovanni.filomeno@poliba.it

**e-mail: tommaso.capurso@poliba.it

***e-mail: marco.torresi@poliba.it

****e-mail: giuseppe.pascazio@poliba.it
} 
Retrofit solutions for existing gas turbines are currently being developed and they can play a crucial role to move the first steps into hydrogen combustion technology. In fact, with minor changes to the actual combustor design, co-firing of hydrogen up to $30 \%$ (by volume fraction) can be achieved, resulting in $11 \%$ of carbon reduction [4]. On-field experience could lead to further development of current gas turbine technology allowing up to $100 \%$ hydrogen firing. Most of existing gas turbines can be retrofitted to either partially or fully burn hydrogen with small modifications, avoiding large capital spending and extending lifetime of existing plants [5].

Even though, green hydrogen is a carbon-free high-energy content vector, its employment in gas turbine combustion gives rise to several issues. Hydrogen flame speed is up to 10 times faster than natural gas, resulting in a higher risk of flashback and consequently damage to the hardware. Hydrogen addition modifies the thermo-acoustic behavior of the combustion system locally, increasing flame temperature, which could lead to higher NOx emissions. Higher auto-ignition risk due to lower ignition delay time should also be accounted. However, hydrogen properties affect also positively the combustion process. Hydrogen addition widens the flammability limits of the fuel, allowing leaner combustion (and thus lower adiabatic flame temperature) with lesser risk of blowout and reduction of NOx emissions.

With the aim to improve the understanding of hydrogen-enriched methane turbulent combustion, a premixed burner tested at the Institut für Verbrennungstechnik, Deutsches Zentrum für Luft-und Raumfahrt (DLR) has been investigated by means of numerical simulations. The burner under study is the well-known PRECCINSTA burner, which has been widely studied both experimentally $[6,7]$ and numerically $[8,13]$. The burner features a swirled stabilized flame, produced by twelve radial vanes downstream of a plenum. In addition to the swirler, a central hub is used to stabilize the flame and hold it attached to the burner. The combustion chamber has a squared cross section, ending into an exhaust duct.

Experiments conducted at DLR showed the effect of hydrogen addition in terms of flame shape, sound pressure and peak frequencies for various operating conditions. In this work, 3D unsteady RANS simulations have been performed for both cold and reacting flows. The numerical model has been assessed against experimental results. First, a 3D unstructured mesh of the burner has been created and a grid independence study (for the cold flow) has been performed. Several combustion models (Species Transport and Partially Premixed) have been used to simulate the coventional methane-air combustion. Finally, a methane-hydrogen fuel blend $\left(20 \% \mathrm{H}_{2}\right.$ by volume) has been simulated at fixed equivalence ratio and thermal power. Flame shapes in terms of $\mathrm{OH}^{*}$ molar concentrations have been compared against experimental $\mathrm{OH}^{*}$ fields obtained by chemiluminescence imaging.

\section{Case study}

The geometry investigated in this work reproduces the premixed swirl-stabilized PRECCINSTA burner (see Fig. 1). The air enters the plenum trough a $25 \mathrm{~mm}$ diameter inlet section and then passes into the swirler, composed by 12 radial vanes. The fuel is injected through 1 $\mathrm{mm}$ orifices in the swirl vanes. Here, the fuel-air premixing takes place and the flow enters the combustion chamber through a burner nozzle with an exit diameter of $D=27.85 \mathrm{~mm}$ and a conical inner bluff body. The combustion chamber has a squared cross-section of $85 \times 85$ $\mathrm{mm}^{2}$ and a height of $114 \mathrm{~mm}$ and ends with a conical surface followed by an exhaust duct with $40 \mathrm{~mm}$ inner diameter.

This burner has been experimentally studied at DLR [14]. In the cited study, flame shapes for various configuration are observed using $\mathrm{OH}^{*}$ line of sight chemiluminescence imaging, a common indicator of heat release. Data were collected for various fuel/air equivalence ratios $(0.70,0.85,1.05), \mathrm{H}_{2}$ volume fraction ( 0 to $40 \%$ in $5 \%$ increments) and thermal power (10, 


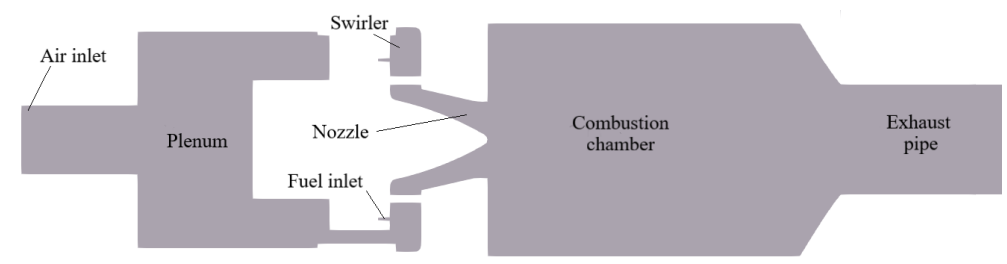

Figure 1. 2D section of the PRECCINSTA premixed swirl burner

$20,30 \mathrm{~kW})$. Herein, the $\phi=0.70, H_{2}$ volume fraction $=20 \%, P_{t h}=20 \mathrm{~kW}$, case has been chosen as reference case for the numerical simulations.

\section{Numerical models and methods}

\subsection{Conservation equation}

Numerical investigations have been conducted in the ANSYS Fluent ${ }^{\circledR}$ environment in order to simulate the experimental work. Herein, unsteady 3D RANS equation have been employed. Continuity and momentum equations can be written in 3D Cartesian coordinates as [15]:

$$
\begin{gathered}
\frac{\partial \rho}{\partial t}+\frac{\partial\left(\rho u_{i}\right)}{\partial x_{i}}=0 \\
\frac{\partial\left(\rho u_{i}\right)}{\partial t}+\frac{\partial\left(\rho u_{i} u_{j}\right)}{\partial x_{j}}=-\frac{\partial p}{\partial x_{i}}+\frac{\partial}{\partial x_{j}}\left[\mu\left(\frac{\partial u_{i}}{\partial x_{j}}+\frac{\partial u_{j}}{\partial x_{i}}-\frac{2}{3} \delta_{i j} \frac{\partial u_{l}}{\partial x_{l}}\right)\right]+\frac{\partial\left(-\rho \overline{u^{\prime} u^{\prime}}\right)}{\partial x_{j}}
\end{gathered}
$$

Here $\rho$ is the density, $p$ is the pressure, $u$ is the velocity, and the subscript $i, j, k$ are the three Cartesian coordinates. The terms on the right hand side of Eq. (2) are respectively the pressure gradient, the divergence of the viscous stress tensor and the divergence of the Reynolds stresses tensor.

The energy equation can be written as:

$$
\rho \frac{D}{D t}\left(e+\frac{1}{2} u_{i}^{2}\right)=\nabla(k \nabla T)+\frac{\partial}{\partial x_{i}}\left(\sigma_{i, j} u_{i}\right)+q
$$

where $e$ is the energy, $k$ the thermal conductivity and $\sigma_{i, j}$ the divergence of the total stresses tensor.

\subsection{Turbulence modeling}

The model used for the turbulence closure of the U-RANS equations is the Realizable $k-\epsilon$ model [16]. The $k$ - $\epsilon$ model [17] is a semi-empirical model based on model transport equations for the turbulence kinetic energy $(k)$ and its dissipation rate $(\epsilon)$. The $k$ - $\epsilon$ models have been widely used in last years to simulate combustion processes where the wall treatment is not a primary concern, thanks to their robustness, economy, and reasonable accuracy [18]. The Realizable $k-\epsilon$ model provides superior performance for flows involving rotation and recirculation [15]. Other turbulence models has been used (i.e. Transition-SST, Reynold Stress, DES-RANS models) but no significant variations in the results have been detected. 


\subsection{Partially premixed combustion model}

Modeling turbulent combustion is one of the most difficult challenges for computational fluid dynamics. Combustion model can be divided into two macro categories: premixed and nonpremixed (or diffusion) combustion.

Premixed combustion models assume that fuel and oxidizer enter the computational domain already perfectly premixed at molecular level. The combustion occurs as a thin flame front that propagates from the burnt gases (products) to the fresh unburnt gases (reactants). The flame front propagation is modeled by solving a transport equation for the densityweighted mean reaction progress variable $c$, which ranges from 0 in the fresh gases to 1 in the burnt gases.

In non-premixed combustion fuel and oxidizer are not mixed before and they enter the computational domain in distinct streams. Mixing must bring reactants in the reaction zone fast enough for combustion to proceed. Under the assumptions of constant thermodynamic pressure and low Mach numbers, heat capacities equal and constant for all species, Lewis numbers all equal to unity [9], the thermochemistry can be reduced to a single parameter: the mixture fraction $f$, defined as

$$
f=\frac{Z-Z_{i, o x}}{Z_{i, f u e l}-Z_{i, o x}}
$$

where $Z_{i}$ is the mass fraction of the generic element $i$. The subscript $o x$ denotes the value at the oxidizer stream inlet and the subscript fuel denotes the value at the fuel stream inlet. Mixture fraction indicates how much of the total mixture comes from the fuel stream.

Under certain hypothesis [9], the mixture fraction method allows to break down the problem into two parts: the mixing problem, consisting in calculating the $f$-field, and the flame structure problem, consisting in linking the temperature, fuel mass fraction and oxidizer mass fraction to $f$. The termochemistry is pre-calculated through a reaction mechanism, the interaction between chemistry and turbulence is modeled using the mixture fraction variance and the informations on temperature, density and species are linked to the mixture fraction $f$ through a Probability Density Function (PDF). The laminar flame speed is calculated through a piecewise-linear polynomial function of $f$ realized to fit results obtained in detailed simulations from previous works [19].

While in theory these two models are sharply distinguished, in practical application they often tends to overlap. In the case study, fuel and oxidizer enter the combustion chamber from two different streams, but the mixing occurs upstream of the combustion chamber. In this work, the Partially Premixed model has been used: it is a combination of the two previous models that solves a transport equation for both the mean reaction progress variable $c$ (to determine the position of the flame front ) and the mean mixture fraction $f$ (and its variance $f^{\prime}$ ). Turbulent flame speed has been calculated through the Zimont model [10-12].

Chemistry can be modeled as being in chemical equilibrium (Equilibrium model), being near chemical equilibrium (Steady Diffusion Flamelet model), or far from chemical equilibrium (Unsteady Laminar Flamelet model).

With the Chemical Equilibrium model, fuel properties are calculated through nonadiabatic equilibrium calculation and they only depends on mean mixture fraction, mixture fraction variance and enthalpy levels. Kinetic effects are not accounted since a reaction mechanism is not present in the model.

The idea behind the steady laminar flamelet approach is the modeling of a turbulent flame brush as an ensemble of discrete, steady laminar flames, called flamelets. The individual flamelets are assumed to have the same structure as laminar flames in simple configurations, and are obtained by experiments or calculations. These laminar flamelets are then embedded 
in a turbulent flame using statistical PDF methods. This formulation takes into account local turbulence effects via strain rates. Thus, the results are not only dependent on the local mixture of fuel and oxidizer and enthalpy levels, but also on the local turbulence level. Laminar flamelet approach allows realistic chemical kinetic effects modeling with considerable computational savings.

Combustion can be modeled as adiabatic or non-adiabatic: adiabatic formulation is a simpler model that involves a two-dimensional look up table but does not allow the modeling of some type of reacting systems (like multiple fuel inlets systems).

In this work, a Partially Premixed model with both Steady Diffusion Flamelet and Chemical Equilibrium modeling of thermochemistry is used. The Steady Diffusion Flamelet model model proved to be the most reliable for this case study.

\subsection{Numerical model}

The cold and reacting flow have been studied by means of 3D unsteady RANS simulations.

Boundary conditions for the cold flow and the methane-air combustion have been collected and deduced from [8]. For the cold flow, a mass flow inlet with $G_{a}=12 \mathrm{~g} / \mathrm{s}$ condition has been imposed on the inlet section. A pressure-outlet condition with atmospheric pressure has been imposed at the outlet and the walls are considered adiabatic and with the no-slip condition. It must be noted that the atmospheric chamber downstream of the exhaust tube is added to push the outlet boundary condition as far as possible from the zone where the mixing and the combustion occurs to avoid the influence of the boundary condition on the results. The methane reacting case is characterized by the same air mass flow rate $\left(G_{a}=12 \mathrm{~g} / \mathrm{s}\right)$, an equivalence ratio of 0.75 , an and a thermal power of $27 \mathrm{~kW}$. Boundary conditions for hydrogen combustion have been inherited by the operating condition reported in [14]. The $\phi=0.70, \mathrm{H}_{2}$ volume fraction $=20 \%, P_{t h}=20 \mathrm{~kW}$, case has been chosen as reference case for the numerical simulations.

The Realizable $k-\epsilon$ model has been used for the turbulence closure of the U-RANS simulations for both cold and reacting flow. The combustion for both methane and hydrogen was modeled with the Partially Premixed combustion model. A time step of $\Delta t=2 \times 10^{-5}$ $s$ was used for all the unsteady simulations. Solution convergence was determined when the residuals was less than $10^{-3}$ for all the variables.

\subsection{Grid independence study}

Before starting the reacting flow simulations, a grid sensitivity analysis has been conducted on two grids by means of unsteady cold flow RANS simulations.

Unstructured meshes with two different grid densities have been created by means of Pointwise ${ }^{\circledR}$ (see Table 1 and Fig. 2). The fine grid has been realized through a refinement of the coarse grid, especially in the mixing zone (between the burner nozzle and the bluff body) and in the first half of the combustion chamber (see Fig. 3).

These grids have been compared to the grid used in [8]. In particular, mean axial and tangential velocity profiles for various sections in the combustion chamber have been compared. It must be noted that in the reference work, LES simulations have been performed thus the grid used is much finer (around 3 millions cells) than the grids created in this study.

Results show good accordance with the reference case (see Figs. 4 and 5). The two grids show a similar trends and the differences on the velocity profile are negligible. However, the fine grid has been chosen because the additional time required for the simulation is negligible and the fine grid may resolve better the mixing process in the reacting flow simulations. 
Table 1. Details of the grids used for the grid sensitivity study

\begin{tabular}{lll}
\hline Parameters & Coarse & Fine \\
\hline n. of cells $\left(\times 10^{6}\right)$ & 1.35 & 1.70 \\
n. of cells on refinement zone $\left(\times 10^{5}\right)$ & 4.25 & 7.80 \\
minimum grid size $[\mathrm{mm}]$ & 2.0 & 1.2 \\
maximum grid size $[\mathrm{mm}]$ & 20 & 20 \\
\hline
\end{tabular}

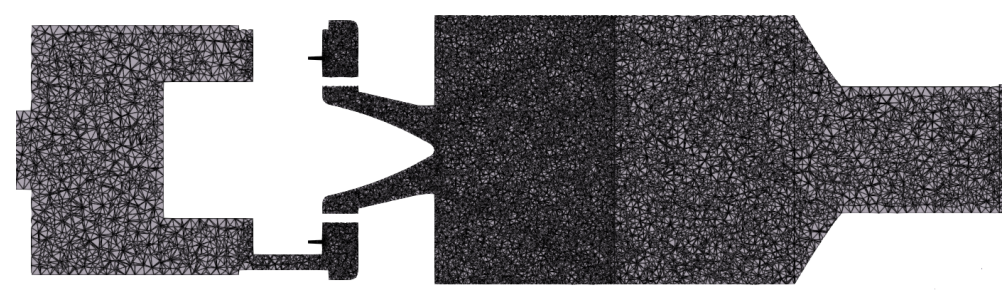

Figure 2. Visualization on a meridian plane of the fine mesh

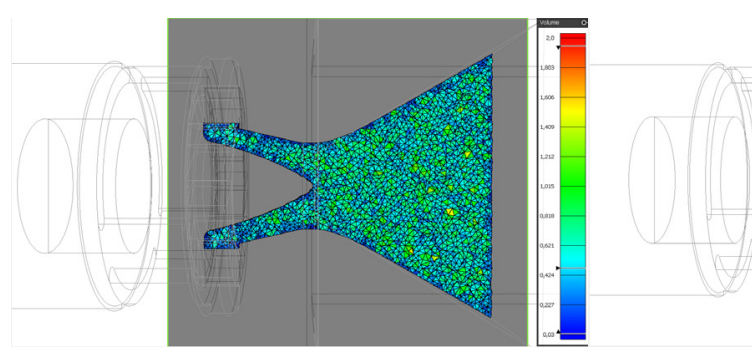

(a)

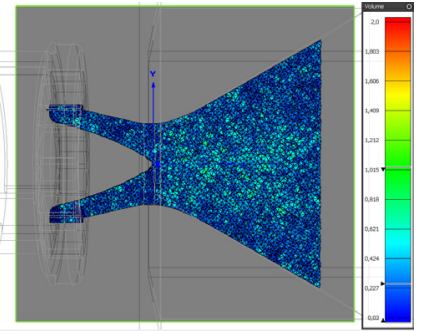

(b)

Figure 3. Cells volume on the refinement zone: (a) Coarse grid (b) Fine grid

The mechanism used to simulate the reaction kinetics for hydrogen combustion is the renowned GriMech 3.0, which provides detailed chemistry, featuring 325 reactions and 53 species [20]. The GriMech mechanism has been already used to study hydrogen-methane flames [21, 22] and it has been validated for a wide range of global fuel equivalence ratios and of hydrogen contents in fuel blends. In order to evaluate the $\mathrm{OH}^{*}$ molar concentration, the reaction mechanism to model the formation and quenching of $\mathrm{OH}^{*}$ used by [23], consisting of 12 reactions ( 2 for formation and 10 for quenching), has been added to the base reaction mechanism.

\section{Results and discussion}

\subsection{Methane combustion}

A campaign of RANS simulations has been performed to investigate the Partially Premixed combustion model and to find the best fitting configuration. This study has been conducted on a methane-air case to compare the results with a reference case simulated by mean of LES simulation, on the same geometry configuration [8].

The first case is a chemical equilibrium, adiabatic simulation. The boundary condition are the ones used in [8] and described in Section 3.4. As stated in Section 3.3, chemical 


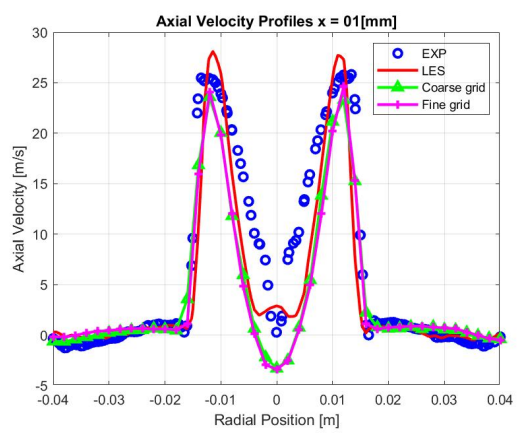

(a)

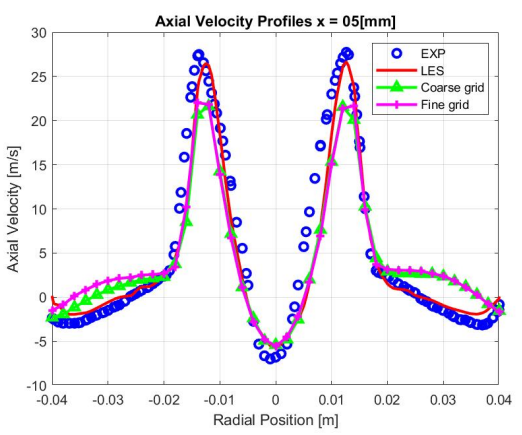

(b)

Figure 4. Axial velocity profiles at $x=1 \mathrm{~mm}$ (a) and $x=5 \mathrm{~mm}$ (b)

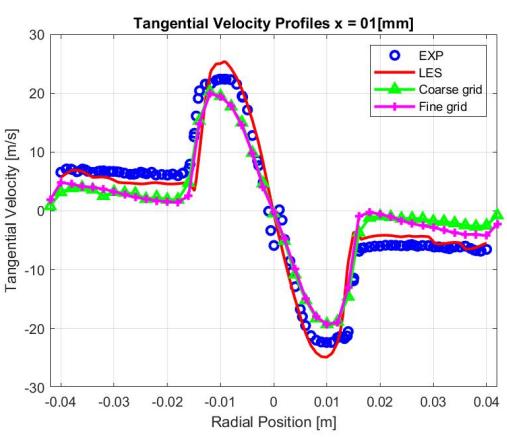

(a)

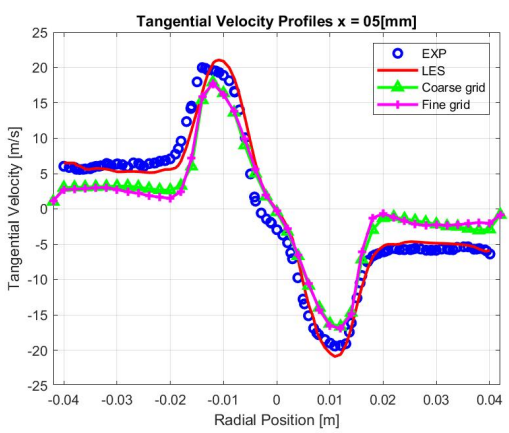

(b)

Figure 5. Tangential velocity profiles at $x=1 \mathrm{~mm}$ (a) and $x=5 \mathrm{~mm}$ (b)

equilibrium model does not account kinetic effects, so this model has been compared to a Steady Laminar Flamelet model. Both cases consider the adiabatic formulation for the energy equation. Results are shown in Fig. 6. Here, mean temperature profiles for two sections in the combustion chamber have been compared. Both models predicts a lower temperature at high radial distance and over-predict it near the symmetry axis. Steady Flamelet model fits better the reference results near to the minima of the profiles.

Different inlets for the fuel are usually an issue for adiabatic models. For this reason, a simulation with steady laminar flamelet model with non-adiabatic formulation has been performed. As it can be seen from Fig. 6, the non adiabatic model fits better the reference results, especially for high radial position. The model under predicts the temperature profiles (mean error is $7.8 \%$ ) but, considering that the reference case results are performed on a much more finer grid and by means of LES simulations, the accuracy is acceptable.

\subsection{Hydrogen addition}

Once the combination of combustion and turbulence models has been chosen (i.e., Partially Premixed Combustion, GriMech 3.0 reaction mechanism with $\mathrm{OH}^{*}$ sub-mechanism [23], Realizable $k-\epsilon$ turbulence model), a simulation with a blended fuel of methane and hydrogen has been carried out. 


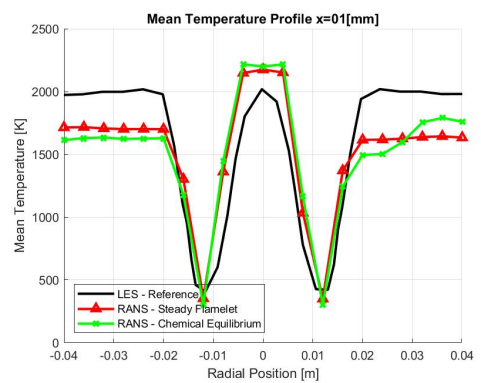

(a)

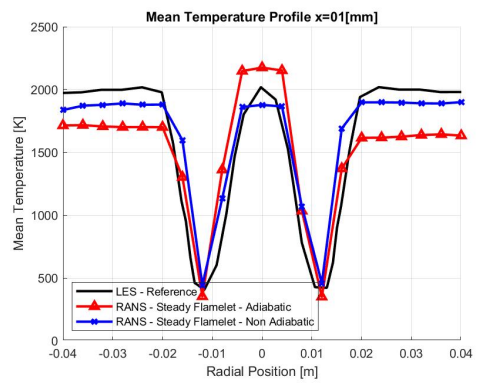

(c)

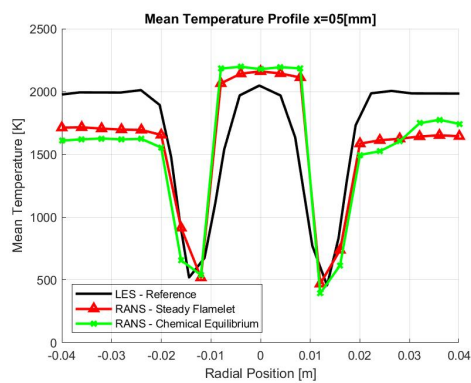

(b)

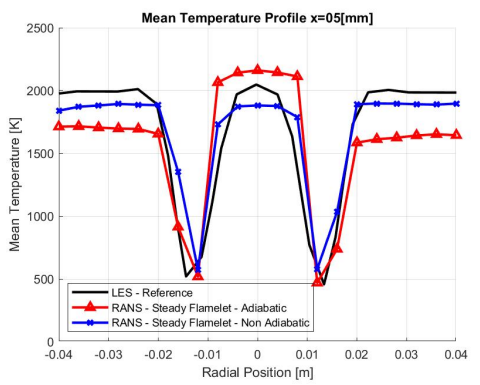

(d)

Figure 6. Mean temperature profile comparison for various sections ( $x=1$ and $x=5 \mathrm{~mm}$ ), for the pure methane combustion simulations

The boundary conditions have been adjusted to match the ones used in the reference experimental work [14], i.e., thermal power $20 \mathrm{~kW}$, equivalence ratio 0.70 and $\mathrm{H}_{2}$ volume fraction $20 \%$. The flame shape assessment has been performed comparing the mean $\mathrm{OH}^{*}$ molar concentration with the experimental line-of-sight $\mathrm{OH}^{*}$ chemiluminescence imaging reported in [14]. As stated in [24], $\mathrm{OH}^{*}$ emission intensity is proportional to $\mathrm{OH}^{*}$ concentration, so the intensity distribution of $\mathrm{OH}^{*}$ chemiluminsecence can be described qualitatively by the $\mathrm{OH}^{*}$ concentration distribution.

In order to post process and compare the experimental flame shape against numerical results, a Matlab ${ }^{\circledR}$ script has been written. Through this script, the $\mathrm{OH}^{*}$ fields picture reported in [14] and in Fig. 7a, has been converted into an indexed gray-scale image (with 256 levels), returned as a numeric array of the same dimensions of the input gray-scale image and compared with the mean $\mathrm{OH}^{*}$ molar concentration profile monitored by Fluent ${ }^{\circledR}$ (normalized by the maximum global value) for several sections of the combustion chamber.

Results for the case with hydrogen addition are shown in Fig. 7. It can be seen that the numerical and experimental profile have the same trend, with a plateau in the central zone and two peaks around 10 and $20 \mathrm{~cm}$ away from the symmetry axis of the combustion chamber. Experimental and numeric peaks positions are close near the chamber inlet while they tend to move away as the distance from the chamber inlet increases.

\section{Conclusions}

Unsteady RANS simulations have been used to investigate the affect of hydrogen addition on the flame shape of a premixed swirled burner and the results were compared to experimental 


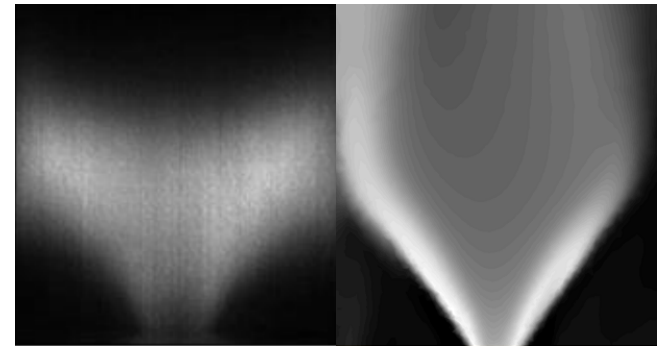

(a)

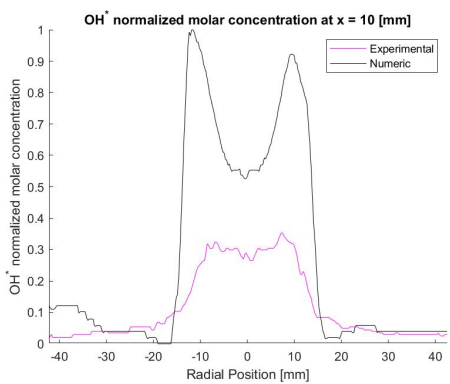

(c)

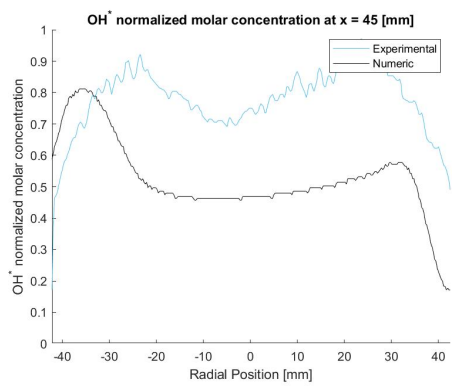

(e) (b)

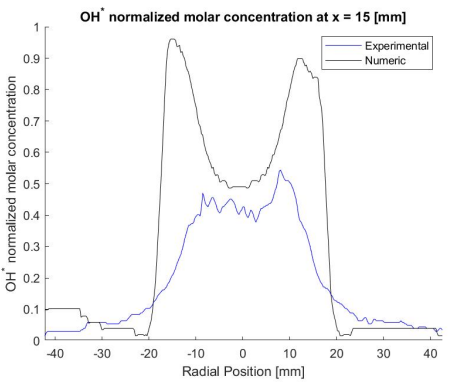

(d)

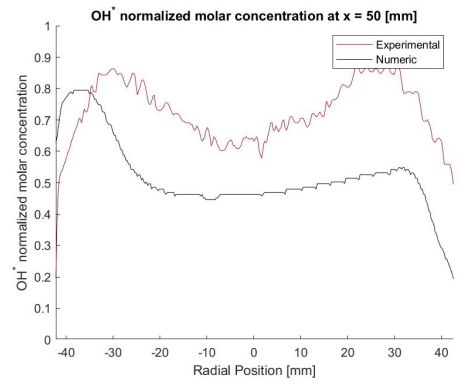

(f)

Figure 7. a) Flame shape by $\mathrm{OH}^{*}$ chemiluminescence imaging from [14]; b) Numerical flame shape by $\mathrm{OH}^{*}$ molar concentration; c) - f) $\mathrm{OH}^{*}$ normalized molar concentration comparisons

data. Mean flow and temperature profile were studied for cold and reacting flow respectively. A comparison between experimental $\mathrm{OH}^{*}$ chemiluminescence imaging and numerical $\mathrm{OH}^{*}$ molar concentration fields have been performed in order to evaluate the flame shape. Results are in good agreement with the experimental data, considering that the experimental flame shape (Fig. 7a) is obtained using time-resolved $\mathrm{OH}^{*}$ chemiluminescence imaging so it is likely to capture the radiation emitted in the whole combustion chamber while the $\mathrm{OH}^{*}$ molar concentration field (Fig. 7b) is evaluated on a single section (parallel to the symmetry axis). While there is an offset, the results showed in Figs. 7c and 7d, exhibit a good accordance with the experimental data. Future studies will be focused on an improvement of the $\mathrm{OH}^{*}$ reaction mechanism and on the post-processing techniques used to link $\mathrm{OH}^{*}$ molar concentration and heat release. Nevertheless, unsteady RANS proved to be a reliable technique for preliminary turbulent premixed combustion analyses. 


\section{Acknowledgements}

The authors gratefully acknowledge W. Meier and the Deutsches Zentrum für Luft-und Raumfahrt (DLR) for providing the detailed geometry of the combustor.

\section{References}

[1] IEA - Global Energy $\mathcal{E} \quad \mathrm{CO} 2$ Status Report 2019, Paris https://www.iea.org/reports/global-energy-co2-status-report-2019 (2019)

[2] European Commission - DG Climate Action: 2030 framework for climate and energy policies, 14 April 2014

[3] T. Capurso, V. Ceglie, F. Fornarelli, M. Torresi, S.M. Camporeale, 75th National ATI Congress, 197, (2020)

[4] The ETN Hydrogen Gas Turbines report - The Path Towards a Zero-Carbon Gas Turbine, Brussels, January 2020

[5] A. Marini, G. Riccio, F. Martelli, S. Sigali, S. Cocchi, ASME Turbo Expo 2010: Power for Land, Sea, and Air, 2, 2010

[6] W. Meier, P. Weigand, X. R. Duan, R. Giezendanner-Thoben, Combust. Flame. 150, 226, (2007)

[7] K. Oberleithner, M. Stöhr, S.H. Im, C.M. Arndt, A.M. Steinberg, Combust. Flame, 162, 3100-3114, (2015)

[8] S. Roux, G. Lartigue, T. Poinsot, U. Meier, C. Bérat, Combust. Flame 141, 40-54, (2005)

[9] T. Poinsot, D. Veynante, Theoretical and Numerical Combustion, (2005)

[10] V. Zimont, Experimental Thermal and Fluid Science, 21, 179-186, (2000).

[11] V. Zimont, W. Polifke, M. Bettelini, and W. Weisenstein, J. of Gas Turbines Power, 120, 526-532 (1998)

[12] V. L. Zimont and A. N. Lipatnikov, Chem. Phys. Reports, 14, 993-1025, (1995)

[13] J. Galpin, A. Naudin, L. Vervisch, C. Angelberger, O. Colin, P. Domingo, Combust. and Flame, 155, 247-266, (2008)

[14] I. Chterev, I. Boxx, Proceedings of the 27th International Colloquium on the Dynamics of Explosions and Reactive Systems (ICDERS), 385, Peking, China, (2019)

[15] ANSYS - Fluent User's Guide v17.1, 2017

[16] T.-H. Shih, W. W. Liou, A. Shabbir, Z. Yang, J. Zhu, Computers Fluids, 24, 227-238, 1995

[17] B.E. Launder, D.B. Spalding, Academic Press, London, England, (1972)

[18] J. P. Wood, Ph.D. thesis, Cranfield University, UK (2004)

[19] J. Gottgens, F. Mauss, N. Peters. Twenty-Fourth Symposium (Int.) on Combustion, Pittsburgh. 24,129-135, (1992)

[20] G.P. Smith, D.M. Golden, M. Frenklach, N.W. Moriarty, B. Eiteneer, M. Goldenberg, C.T. Bowman, R.K. Hanson, S. Song, W. C. Gardiner Jr., V.V. Lissianski and Z. Qin, GriMech 3.0 (1999)

[21] J.Y. Ren, W. Qin, F. Egolfopoulos, T. Tsotsis, Combust. and Flame, 124, 717-720, (2001)

[22] J.L. Gauducheau, B. Denet, G. Searby, Combustion Science and Technology, 137, 8199, (1998)

[23] H. Lei, Y. Gong, Combust. and Flame, 201, 12-22, (2019)

[24] D. Giassi, S. Cao, B. A. V. Bennett, D. P. Stocker, F. Takahashi, M. D. Smooke, M. B. Long, Combust. and Flame, 167, 198-206, (2016) 